\title{
Magneto-optical coupling in bilayer composites of Terfenol-D and Polycarbonate
}

\author{
Luo Xiaobin $\cdot$ Wu Tianxiang $\cdot$ Zhang Ying $\cdot$ \\ Zhang Ning
}

Received: 20 July 2013/ Accepted: 29 October 2013/Published online: 21 November 2013

(C) The Author(s) 2013. This article is published with open access at Springerlink.com

\begin{abstract}
Bilayer composites that can show magnetobirefringence effect were fabricated based on the product effects of magnetostriction and stress-birefringence. Large magneto-optical responses were obtained under a magnetic field lower than $700 \mathrm{Oe}$ at room temperature for the bilayers. The magneto-optical retardation was observed to decrease along the direction vertical to the interface, suggesting a non-uniform stress distribution along the thickness direction in the elasto-optical layer. Local action principle or Saint-Venant's principle was taken into account in analysis of the interlayer elastic coupling and the magneto-birefringence effect. The theory that was consistent with experiments was obtained.
\end{abstract}

\section{Introduction}

Magneto-birefringence (MB) effect has being attracted sustained attention due to its potential applications in the fields of magnetism-light conversion and magneto-optical modulation [1-3]. In 1960s, some semiconductor films were found to show MB effects, but only at lower temperature (no higher than liquid nitrogen temperature) and with stronger magnetic field (higher than thousands Oe) [2, 3]. Recently, the MB response obtained in laminate composites with lower magnetic field and at room temperature makes it possible to get applications of MB effect [4].

Most layered composites play the role through the interfacial elastic coupling. The stress-inducing layer, such

L. Xiaobin $\cdot$ W. Tianxiang $\cdot$ Z. Ying $\cdot$ Z. Ning $(\bowtie)$ Magnetoelectronic Lab, Nanjing Normal University and Jiangsu Key Laboratory On Opto-Electronic Technology,

Nanjing 210023, China

e-mail: zhangning@njnu.edu.cn as the magnetostrictive or electrostrictive layer, usually brings a force parallel to the interface on the surface of the elastic layer connected. So far, it tends to think that the stress induced by the surface force distributes uniformly along the thickness direction in the elastic layer when discussing the effect of interlayer elastic coupling [5, 6]. However, this supposition often gives rise to a theory that does not match experimental results. For example, the electro-optical retardation observed decreases with increasing the distance from the beam incident point to the interface in a laminated composite of electrostriction and elasto-optical medium. But the theory under the supposition gives an invariant electro-optical retardation in thickness direction [7]. Obviously, it is necessary to reconsider the presumption that "stress distributes uniformly along the thick direction". In fact, according to the principle of local action, namely, Saint-Venant's principle [8], under a surface force, the stress in an elastic medium distributes nonuniformly in the direction perpendicular to the surface.

According to Saint-Venant's principle "in elastic bodies, the stress caused by a load distributed on a small area (or volume) relates only to the resultant force and moment of the load in the region far from the loading zone". When solving specific problems, if the stresses cared are only at the area far from the loading zone, under the conditions of keeping the resultant force and moment invariant, we can use an equivalent load that distributed uniformly according to the convenience of computation or experiment. But more careful consideration must be given near the loading area. The region near the interface of a laminate composite is just located near the loading area, therefore, there may exist non-uniform stress distribution. But, so far, the law of the stress distribution along the direction vertical to the action line is unclear, because the Saint-Venant principle has not been thoroughly proven yet in mathematics, or the 
principle of local action cannot be quantified since the perfect boundary conditions are difficult to get $[9,10]$. However, we found, after making some reasonable approximation, a complete set of boundary conditions can be given, and the corresponding stress distribution can be obtained for planar layered composite materials.

\section{Experimental results and discussion}

The samples under investigation are layered composites of magnetostrictive and elasto-optical materials. Elasto-optical materials are those being capable of displaying stressbirefringence effects. When polarized light passes through these materials subjected to a stress, it separates into two light vectors, which often noted as ordinary $(o)$ and the extraordinary $(e)$ lights, respectively, traveling at different velocities. Each of the lights orients parallel to the direction of principal stresses, but perpendicular to each other, resulting in two different indices of refraction, which vary as a function of the stress applied [11, 12].

Polycarbonate (PC) is an easy-gained polymer with high transparency, optical isotropy and no natural birefringence without inner stress. It was often used in the study of photoelasticity due to its strong effect of stress-birefringence and very small optical and mechanical creep at room temperature $[13,14]$.

The phase difference between the transmitted $o$ and $e$ lights from a birefringence medium is known as retardation $\delta$, which can be expressed as $[11,15]$

$\delta=2 \pi c d \sigma / \lambda$

where $\sigma$ is the difference of principal stresses, $d$ is the distance that the beam passed through, $c$ is the stressoptical constant and $\lambda$ is the wave length of the incident light.

PC plates of $5 \mathrm{~mm}$ in width were cut into strips of $20 \mathrm{~mm}$ in length and $2.75 \mathrm{~mm}$ in thickness. Residual stress that perhaps induced in the primary manufacturing process in PC samples should be relieved through annealing before use. For the purpose, all the PC samples were heated to $158{ }^{\circ} \mathrm{C}$, and kept hot for $2 \mathrm{~h}$, then cooled to $140{ }^{\circ} \mathrm{C}$ at the rate of $5{ }^{\circ} \mathrm{C} / \mathrm{h}$, and furnace cooled. The stress-birefringence test performed with the PC strip used reveals the stressoptical constant $c=70.98 \times 10^{-12} / \mathrm{Pa}$ [16].

Rare earth-iron alloy terbium dysprosium iron $\mathrm{Tb}_{1-}$ ${ }_{x} \mathrm{Dy}_{x} \mathrm{Fe}_{2-y}$ (Terfenol-D or TDF) is well known for its great performance in magnetostriction [17-19]. Its magnetostrictive coefficients can reach 1,500-1,800 ppm under stress, which is much larger than that without stress due the so-called "jump-effect" [20]. Commercial TDF strips with the sizes of $20 \times 5 \times 2 \mathrm{~mm}^{3}$ were employed as the magnetostrictive phase and bonded to PC strip(s) with super glue to form layered composites, as shown in Fig. 1a. Their greatest magnetostriction was directed to the length, the $y$ direction, as shown in Fig. 1b. The TDF in the composite should be considered to work under stress due to the retroaction from the PC layer.

MB response with the bilayers of TDF/PC was measured. A laser of $632.8 \mathrm{~nm}$ in wave length, a set of polariser and analyzer, as well as a digital Lux meter (Tondaj LX1010B) were used in the experiment. Figure 2 shows the MB response at different point $z$ in the PC layer for the $\mathrm{TDF} / \mathrm{PC}$ composite with the TDF in a thickness of $2 \mathrm{~mm}$,

(a)
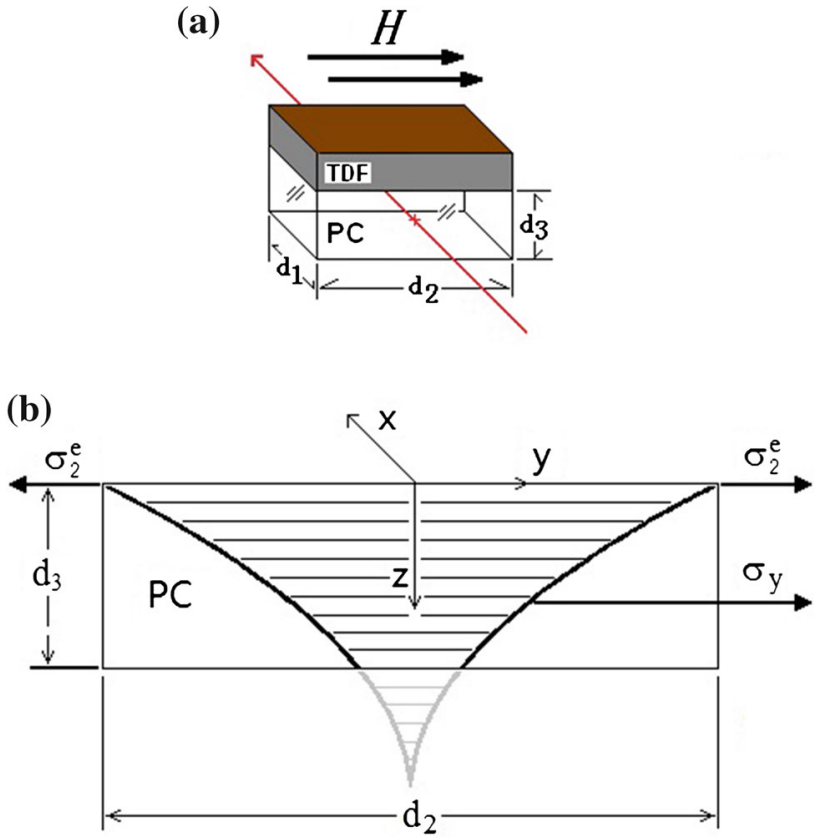

Fig. 1 The schematic diagram of a bilayer composite of MB effect (a), and the external load subjected, as well as the internal stress distribution (b)

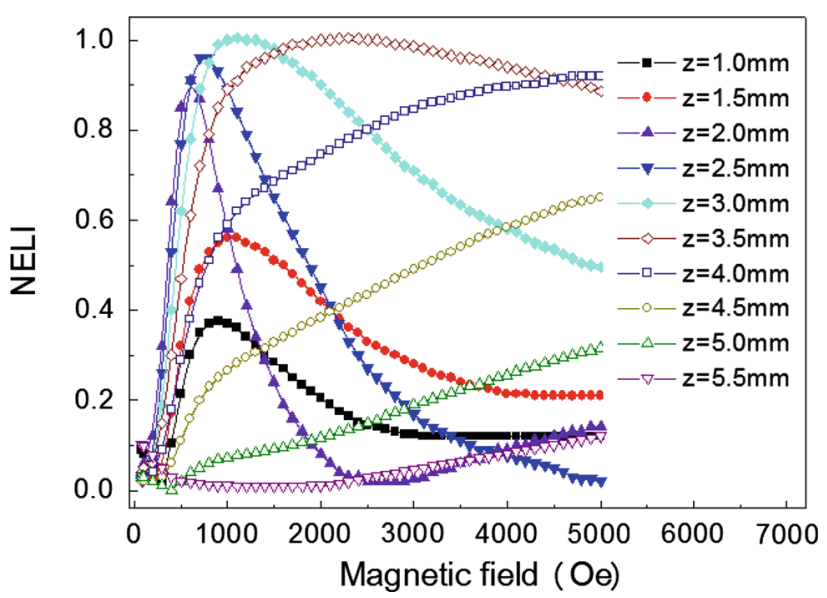

Fig. 2 The MB response at different point of the PC layer in the TDF/PC composite with the TDF in thickness of $2 \mathrm{~mm}$, where $x$ is the distance from the points to the interface 
where $z$ is the distance from the point measured to the interface; NELI means the normalized emergent light intensity, which equals to $I_{2} / I_{2 \max }$, where $I_{2}$ is the emergent light intensity measured, and $I_{2 \max }$ is the maximum value of $I_{2}$. The size of the PC layer in the composite is $20 \times 5 \times 6.9 \mathrm{~mm}^{3}$. It is found that, with increasing $z$, the (magneto-optical) retardation increases first, and reaches its maximum value at $z=0.2 \mathrm{~mm}$, then decreases. Second, a MB response of reverse direction was observed when $z$ exceeds $5.0 \mathrm{~mm}$, suggesting a bending behavior in the composite with increasing magnetic field.

Figure 3 shows the MB response at different point of the PC layer in the TDF/PC bilayer with a layer of TDF of $4 \mathrm{~mm}$ in thickness. It is found that the retardation increases monotonously with increasing magnetic field and decreases with increasing the distance to the interface. No MB of reverse direction is observed, meaning that no bending

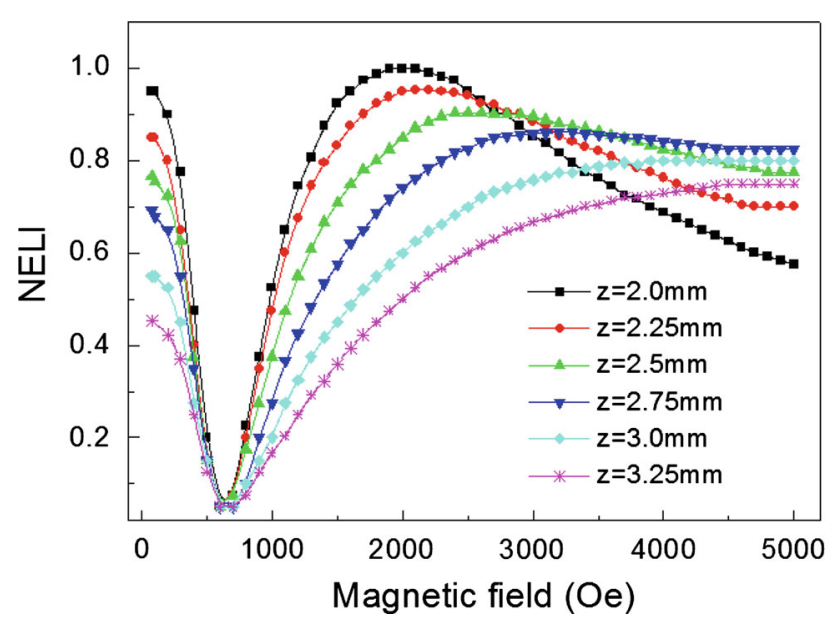

Fig. 3 The MB response at different point of the PC layer in the $\mathrm{TDF} / \mathrm{PC}$ composite with the TDF in thickness of $4 \mathrm{~mm}$, where $x$ is the distance from the points to the interface

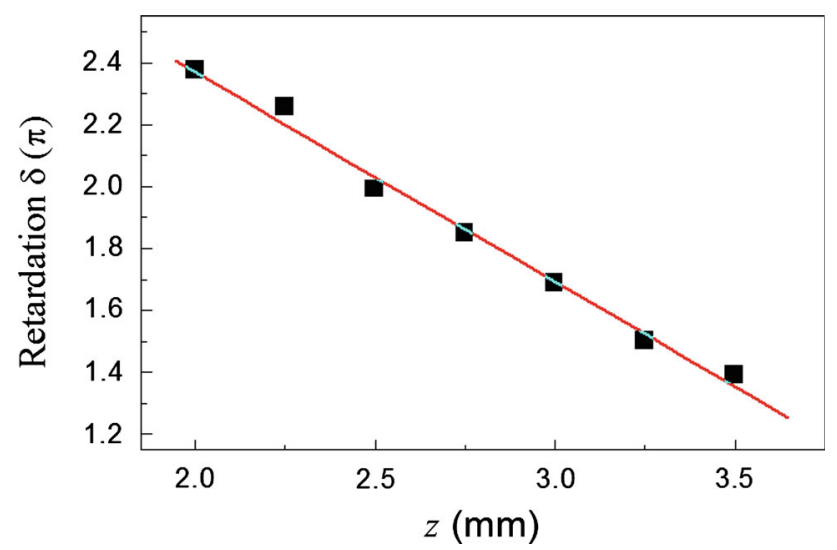

Fig. 4 The retardation as a function of the distance to the interface in the $\mathrm{PC}$ layer of a TDF/PC composite with the PC layer in size of $20 \times 5 \times 6.9 \mathrm{~mm}^{3}$ occurred in the composite with a thick enough layer of TDF since TDF has a higher bending strength.

According to Fig. 3, we can assess the corresponding retardations for each curve, respectively. The retardation as a function of $z$ is shown in Fig. 4, where the points are the retardations estimated from Fig. 3, and the line is the fitted curve, which presents a straight line.

\section{Theoretical estimation of the MB effect for bilayer magnet/elasto-optical composite}

For a free-body layered structure, assuming that elastic coupling at the interface was ideal, namely, any flexural deformations of the layers can be ignored, and the magnetostriction of the ferromagnetic phase is along the direction of the length of the sample, the constitutive equations for the ferromagnetic phase are given by

$$
\begin{aligned}
{\left[\begin{array}{l}
S_{1}^{\mathrm{m}} \\
S_{2}^{\mathrm{m}} \\
S_{3}^{\mathrm{m}}
\end{array}\right]=} & {\left[\begin{array}{lll}
s_{11}^{\mathrm{m}} & s_{12}^{\mathrm{m}} & s_{13}^{\mathrm{m}} \\
s_{21}^{\mathrm{m}} & s_{22}^{\mathrm{m}} & s_{23}^{\mathrm{m}} \\
s_{31}^{\mathrm{m}} & s_{32}^{\mathrm{m}} & s_{33}^{\mathrm{m}}
\end{array}\right]\left[\begin{array}{l}
\sigma_{1}^{\mathrm{m}} \\
\sigma_{2}^{\mathrm{m}} \\
\sigma_{3}^{\mathrm{m}}
\end{array}\right] } \\
& +\left[\begin{array}{lll}
q_{11}^{\mathrm{m}} & q_{12}^{\mathrm{m}} & q_{13}^{\mathrm{m}} \\
q_{21}^{\mathrm{m}} & q_{22}^{\mathrm{m}} & q_{23}^{\mathrm{m}} \\
q_{31}^{\mathrm{m}} & q_{32}^{\mathrm{m}} & q_{33}^{\mathrm{m}}
\end{array}\right]\left[\begin{array}{l}
H_{1} \\
H_{2} \\
H_{3}
\end{array}\right],
\end{aligned}
$$

where $H_{i}$ is the magnetic field, $H_{1}=H$ and $H_{2}=H_{3}=0$ in the present case, $S_{i}^{\mathrm{m}}, s_{i j}^{\mathrm{m}}, q_{i j}^{\mathrm{m}}$ and $\sigma_{i}^{\mathrm{m}}(i, j=1,2,3)$ are the strains, compliances, piezomagnetic coefficients $\left(q_{i j}^{\mathrm{m}}=\partial l_{i j}^{\mathrm{m}} / \partial H_{i}\right.$ and $\left.q_{21}^{\mathrm{m}}=q_{12}^{\mathrm{m}}\right)$ and the stresses in different directions of the ferromagnetic layer, respectively, $l_{i j}^{\mathrm{m}}$ is the magnetostrictive coefficient, the superscript " $\mathrm{m}$ " means the ferromagnetic phase and the subscript $i(j)=1,2,3$ indicates different directions. While, the stress-strain equations in the elasticoptical phase can be written as:

$$
\left[\begin{array}{c}
S_{1}^{\mathrm{e}} \\
S_{2}^{\mathrm{e}} \\
S_{3}^{\mathrm{e}}
\end{array}\right]=\frac{1}{E} \times\left[\begin{array}{ccc}
1 & -\mu & -\mu \\
-\mu & 1 & -\mu \\
-\mu & -\mu & 1
\end{array}\right]\left[\begin{array}{c}
\sigma_{1}^{\mathrm{e}} \\
\sigma_{2}^{\mathrm{e}} \\
\sigma_{3}^{\mathrm{e}}
\end{array}\right],
$$

where $E, \mu$ and $\sigma_{i}^{\mathrm{e}}$ are Young's modulus, Poisson's ratio and stress of elastic-optical phase, respectively, the superscript "e" means the elasto-optical phase. For ideal interfacial coupling, there are following boundary conditions $\sigma_{3}^{\mathrm{e}}=\sigma_{3}^{\mathrm{m}}=0, S_{1}^{\mathrm{e}}=S_{1}^{\mathrm{m}}, S_{2}^{\mathrm{e}}=k S_{2}^{\mathrm{m}}, \sigma_{1}^{\mathrm{e}}(1-v)-$ $\sigma_{1}^{\mathrm{m}} v=0 \quad$ and $\quad \sigma_{2}^{\mathrm{e}}(1-v)-\sigma_{2}^{\mathrm{m}} v=0$, where $\quad v=$ $v^{\mathrm{m}} /\left(v^{\mathrm{e}}+v^{\mathrm{m}}\right)$ and $1-v$ are the volume fractions of the ferromagnet and elasto-optical phases, respectively. In addition, there are following relations $s_{11}^{\mathrm{m}}=s_{22}^{\mathrm{m}}$ and $s_{12}^{\mathrm{m}}=$ $s_{21}^{\mathrm{m}}$ for an isotropy elasto-optical layer. Combining Eqs. 2 and 3 leads to 


$$
\begin{aligned}
\sigma_{1}^{\mathrm{e}} & =\frac{\left(1-E s_{11} v^{\prime}\right) q_{11}+\left(\mu+E s_{12} v^{\prime}\right) q_{21}}{\left(1-E s_{11} v^{\prime}\right)^{2}-\left(\mu+E s_{12} v^{\prime}\right)^{2}} \mathrm{EH} \\
\sigma_{2}^{\mathrm{e}} & =\frac{\left(\mu+E s_{12} v^{\prime}\right) q_{11}+\left(1-E s_{11} v^{\prime}\right) q_{21}}{\left(1-E s_{11} v^{\prime}\right)^{2}-\left(\mu+E s_{12} v^{\prime}\right)^{2}} \mathrm{EH},
\end{aligned}
$$

where $\quad s_{11}=s_{11}^{\mathrm{m}}, \quad s_{12}=s_{12}^{\mathrm{m}}, \quad q_{11}=q_{11}^{m}, \quad q_{21}=q_{21}^{m}$, $v^{\prime}=(1-v) / v$. Since piezomagnetic coefficient $q_{11}$ is often much greater than $q_{21}$ for a ferromagnet, we can prove that $\sigma_{1}^{\mathrm{e}}>>\sigma_{2}^{\mathrm{e}}$ from Eq. 4. Thus, $\sigma_{2}^{\mathrm{e}}$ can be ignored, and $\sigma_{1}^{\mathrm{e}}$ is just the stress acted on and parallel to the surface of the PC layer.

Assuming (1) ideal elastic coupling forms in the interlayer of a tabulate bilayer composite, (2) the elastic medium layer only bears a tensile stress of single side $\sigma_{1}^{\mathrm{e}}$ that is at and parallel to the interface, that is the y direction, (3) the tensile stress generates deformations $\Delta d_{i}$ and $\Delta d_{\mathrm{f}}$ in $y$ direction at the interface and free surface, respectively, for the elastic layer. Thus, based on the considerations above, the stress and strain in the elastic layer can be considered as a plane problem. According to the elasticity, if all body forces are zero, we have

$\sigma_{z}=\frac{\partial^{2} \varphi}{\partial y^{2}}, \sigma_{y}=\frac{\partial^{2} \varphi}{\partial z^{2}}, \tau_{z y}=-\frac{\partial^{2} \varphi}{\partial z \partial y}$,

where $\varphi$ is the stress function for the plane. Meanwhile, the stress function should also meet the biharmonic equation as below

$\nabla^{4} \varphi=\left(\frac{\partial^{2}}{\partial z^{2}}+\frac{\partial^{2}}{\partial y^{2}}\right)\left(\frac{\partial^{2}}{\partial z^{2}}+\frac{\partial^{2}}{\partial y^{2}}\right) \varphi=0$.

Considering that the external load directs only to the $y$ direction, the tensile stress in thick direction $z$ can be neglected, namely, $\sigma_{z}=\partial^{2} \varphi / \partial y^{2}=0$.

For the flat elastic medium and the stress subjected as shown in Fig. 1b, we can list the corresponding boundary conditions as below

$$
\begin{aligned}
& (v)_{z=0, y=d_{2} / 2}=\Delta d_{i}, \\
& (v)_{z=d_{3}, y=d_{2} / 2}=\Delta d_{\mathrm{f}},
\end{aligned}
$$

where $v$ and $y$ are the displacements in $y$ and $z$ directions, respectively, $\Delta d_{i}$ and $\Delta d_{\mathrm{f}}$ are the strains of $\mathrm{PC}$ at interface and free surface, respectively.

That the principal stress in $z$ direction equals to zero leads to

$\frac{1}{d_{3}} \int_{0}^{d_{3}}\left(\Sigma_{y}+T_{z y}\right)_{y=d_{2} / 2} \mathrm{~d} z+\frac{d_{2}}{2} \sigma_{1}^{\mathrm{e}}=0$,

That the principal stress in $y$ direction equals to zero results in $\frac{2}{d_{2}} \int_{0}^{d_{2} / 2}\left(T_{y z}\right)_{z=d_{3}} \mathrm{~d} y=0$

And that principal moment equals to zero yields,

$$
\begin{aligned}
& \frac{1}{d_{3}} \int_{d_{3}}^{0}\left(d_{3}-z\right)\left(\Sigma_{y}+T_{z y}\right)_{y=d_{2} / 2} \mathrm{~d} z+\frac{2}{d_{2}} \int_{0}^{d_{2} / 2} y\left(T_{y z}\right)_{y=d_{3}} \mathrm{~d} y \\
& \quad+\frac{d_{2}}{2} d_{3} \sigma_{1}^{\mathrm{e}}=0
\end{aligned}
$$

where $\Sigma_{y}=\frac{1}{y} \int_{0}^{y} \sigma_{y} \mathrm{~d} y, T_{z y}=\frac{1}{y} \int_{0}^{y} \tau_{z y} \mathrm{~d} y$ and $T_{y z}=\frac{1}{z} \int_{0}^{z} \tau_{y z} \mathrm{~d} z$ are the sum of the tensile and shear stresses in $y$ and $z$ directions, respectively. In addition, that shear stress is zero at the free surface gives,

$\left(\tau_{z y}\right)_{z=d_{3}}=0$

According to Saint-Venant principle, the sphere of influence of a local load is limited [8, 9]. Assuming the scope of the influence in $z$ direction is $h$, then all the stress is zero when $z \geq h$, thus,

$\left(\sigma_{y}\right)_{z=h}=0$,

Combining Eqs. 4-13 leads to

$\sigma_{y}=\left[\frac{8}{d_{3}^{2}}\left(\frac{E \Delta d_{\mathrm{f}}}{d_{2}}+\frac{5 d_{2}}{2} \sigma_{1}^{\mathrm{e}}\right)\left(y-\frac{d_{2}}{4}-\frac{d_{3}}{6}\right)+\frac{3 d_{2} \sigma_{1}^{\mathrm{e}}}{d_{3}}\right]\left(2 d_{3}-3 z\right)$

$\tau_{z y}=\frac{4}{d_{3}^{2}}\left(\frac{E \Delta d_{\mathrm{f}}}{d_{2}}+\frac{5 d_{2}}{2} \sigma_{1}^{\mathrm{e}}\right)\left(3 z^{2}-4 d_{3} z+d_{3}^{2}\right)$,

where $\Delta d_{\mathrm{f}}=\frac{d_{2} G l_{11}^{\mathrm{m}}}{2 E-G}-\frac{d_{2}^{2} \sigma_{1}^{\mathrm{e}}(10 E-G)}{2 E(2 E-G)}$, and $\sigma_{1}^{\mathrm{e}}$ can be obtained from Eq. 4.

It can be seen from the results above, (1) the tensile and shear stresses vary in direction $z$ follow a linear and a point conic functions, respectively, (2) both stresses relate not only to the external load but also to the nature of the materials involved. Since the magneto-optical retardation, which is proportional to the stress loaded (see Eq. 1), linearly decayed in the thickness direction of the PC layer, as shown in Fig. 4, we can reach the conclusion that the tensile stress is the principle stress acted on PC layer. Thus, according to Eq. 1, the magneto-optical retardation can be rewritten as

$\delta=\frac{2 \pi c d_{1}}{\lambda}\left[\frac{8}{d_{3}^{2}}\left(\frac{E \Delta d_{f}}{d_{2}}+\frac{5 d_{2}}{2} \sigma_{1}^{\mathrm{e}}\right)\left(y-\frac{d_{2}}{4}-\frac{d_{3}}{6}\right)+\frac{3 d_{2} \sigma_{1}^{\mathrm{e}}}{d_{3}}\right]\left(2 d_{3}-3 z\right)$,

Setting the polarization direction of both polarizers perpendicular to each other and the laser beam passes 


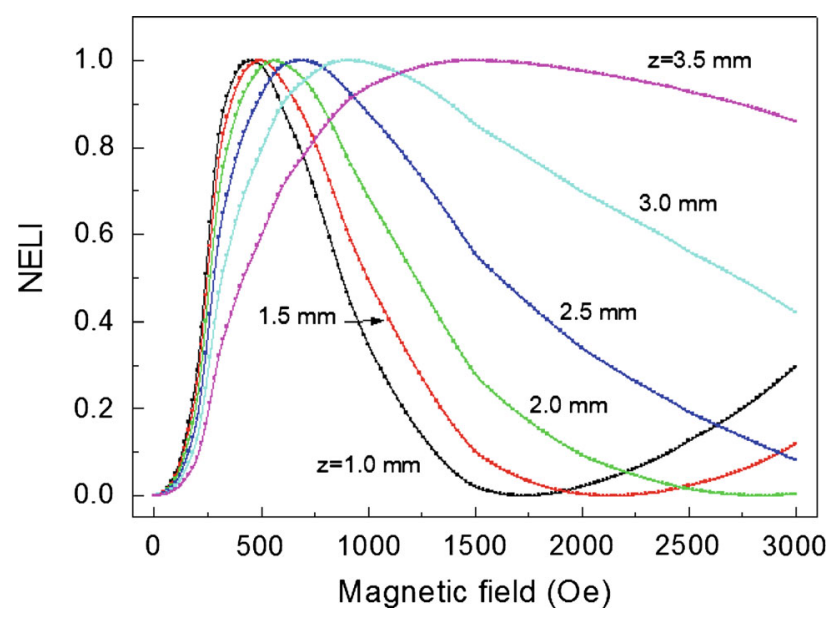

Fig. 5 Calculated curves of NELI versus magnetic field from Eq. 16 with the laser beam incidence at different point of $z$ for the sample with the sizes of PC layer in $d_{1} \times d_{2} \times d_{3}=6.3 \times 20 \times 6.9 \mathrm{~mm}^{3}$ and TDF layer in $6.3 \times 20 \times 4.0 \mathrm{~mm}^{3}$, respectively

through the middle point in $y$ direction $(y=0)$ for the PC layer, we consequently obtain the normalized intensity of emergent light as below

$$
\begin{aligned}
& \text { NELI }=\sin ^{2}\left(\frac{\delta}{2}\right) \\
& =\sin ^{2}\left\{\frac{\pi c d_{1}}{\lambda d_{3}^{2}}\left[\frac{4 E \Delta d_{\mathrm{f}}}{d_{2}}\left(\frac{d_{2}}{2}+\frac{d_{3}}{3}\right)+d_{2} \sigma_{1}^{\mathrm{e}}\left(5 d_{2}+\frac{d_{3}}{3}\right)\right]\left(3 z-2 d_{3}\right)\right\} .
\end{aligned}
$$

Equation 16 indicates that the emergent light intensity not only relies on applied field, the physical parameters of the materials involved and the wave length of the incident light, but also on the geometrical sizes of the composite, suggesting that the MB effect in layered composites is highly size-sensitive. From Eq. 16, we can work out the curves of BR response with laser beam incidence at different point $z$, as shown in Fig. 5. All the parameters used in the calculation are $d_{1} \times d_{2} \times d_{3}=6.3 \times$ $20 \times 6.9 \mathrm{~mm}^{3}, \quad c=70.98 \times 10^{-12} / \mathrm{Pa}, \quad \lambda=632.8 \mathrm{~nm}$, $E=2,430 \times 10^{6} \mathrm{~N} / \mathrm{m}^{2}, \quad G=E / 2(1+\mu), \quad \mu=0.38$, $s_{11}=125 \times 10^{-12} \mathrm{~m}^{2} / \mathrm{N}$ and $s_{12}=-17 \times 10^{-12} \mathrm{~m}^{2} / \mathrm{N}$, respectively. Comparing Fig. 5 with Fig. 3, we find that the retardation for both groups of curves corresponding to the same incident point $z$ is basically the same except some difference in initial phase, which always exists for a practical PC layer due to the possible remanent stress produced in the process of preparation. Thus, Fig. 5 suggests that the theoretical analysis above is reasonable considering that it is difficult to set a suitable initial phase in theoretical derivation.

\section{Conclusion}

Layered composites of ferromagnet and elasto-optical material can show a magneto-birefringence (MB) effect that can work at room temperature and lower magnetic field. These characters make the layered MB composites be superior to the magneto-optical semiconductors found so far. The MB effect in a bilayer composite is highly sizesensitive and position-sensitive since the elasto-optical layer was acted by a unilateral mechanical stress. The stress in the direction parallel to the interface linearly decreases, so the magneto-optical retardation also linearly declines along the thickness direction of the elasto-optical layer.

Acknowledgments This work was supported by the National Science Foundation of China under Grant No. 51277098.

Open Access This article is distributed under the terms of the Creative Commons Attribution License which permits any use, distribution, and reproduction in any medium, provided the original author(s) and the source are credited.

\section{References}

1. S. Teitler, E.D. Palik, Phys. Rev. Lett. 5, 546 (1960)

2. P. S. Pershan, J. Appl. Phys. 38(3) 1482 (1967)

3. P.H. Lissberger, M.R. Parker, J. Appl. Phys. 42, 1708 (1971)

4. X.B. Luo, D. Wu, N. Zhang, J. Appl. Phys. 113, 173903 (2013)

5. I.J. Busch-Vishniac, Phys. Today 51, 28 (1998)

6. N. Fujimura, T. Ishida, T. Yoshimura, T. Ito, Appl. Phys. Lett. 69, 1011 (1996)

7. L. Xiang-Yang, Z. Ning, L. Xiao-Bin, W. Wei, W. Dong, G. JianSen, Chin. Phys. B 20(3) 037802 (2011)

8. R.A. Toupin, Arch. Rat. Mech. Anal. 18, 83 (1965)

9. R.A. Toupin, Trans. N. Y. Acad Sci. 28, 221-232 (1965)

10. J. K. Knowles, Arch. Rat. Mech. Anal. 211-22(1966)

11. Y.N. Ning, T.Y. Liu, D.A. Jackson, Rev. Sci. Instrum. 63(2), $5771(1992)$

12. K. P. Koo, G. H. Sigel, IEEE J Quantum Electronics 18(4), 670 (1982)

13. A. Baldi, F. Bertolino, F. Ginesu, Optics and Lasers in Engineering 45(5), 612 (2007)

14. R. H. Blakemore, B. Marsden, Strain 16(3), 124 (1980)

15. C. Pappalettre, U. Galietti, Strain 31(2), 69 (1995)

16. X.-B. Luo, D. Wu, N. Zhang, Chin. Phys. Lett. 30(7) 077801 (2013)

17. H.T. Savage, R. Abbundi, A.E. Clark, O.D. McMasters, J. Appl. Phys. 50(B3), 1674 (1979)

18. K.R. Dhilsha, G. Markandeyulu, Rao K. V.S. Rama, J. Appl. Phys. 70(8), 4450 (1991)

19. M. Ciria, J. I. Arnaudas, A. del Moral, G. J. Tomka, C. de la Fuente, P. A. J. de Groot, M. R. Wells, R. C. C. Ward, Phys. Rev. Lett. 75(8), 1634(1995)

20. A. E. Clark, J. P. Teter, O. D. McMasters, J. Appl. Phys. 63(8), 3910 (1988) 